\title{
Importance of risk factors for the evaluation of patients with a suspected pulmonary embolism
}

\author{
JOACHIM GRUETTNER $^{1 *}$, TIM VIERGUTZ $^{2 *}$, MERLE BOLTE $^{1}$, THOMAS HENZLER $^{3}$, \\ STEFAN O. SCHOENBERG ${ }^{3}$, SONJA SUDARSKI ${ }^{3}$, PAUL APFALTRER ${ }^{3}$ and THOMAS WALTER ${ }^{1}$ \\ ${ }^{1}$ Emergency Department; ${ }^{2}$ Clinic for Anesthesiology and Operative Intensive Medicine; ${ }^{3}$ Institute of Clinical Radiology \\ and Nuclear Medicine, University Medical Center Mannheim, Medical Faculty Mannheim, \\ Heidelberg University, Mannheim, Baden-Württemberg D-68167, Germany
}

Received July 4, 2014; Accepted March 11, 2015

DOI: 10.3892/etm.2015.2395

\begin{abstract}
The reliable exclusion of a pulmonary embolism (PE) in hemodynamically stable patients remains a challenge. The European Society of Cardiology guidelines for PE diagnosis published in 2008 and updated in 2014 recommend a lowthreshold computed tomography (CT) indication for patients with a high probability of pulmonary embolism or those with elevated levels of D-dimers. Certain elements of the recommendations are controversial, while others, including the evaluation of the risk factors for PE, are considered only in individual cases. In the present study, various risk factors, including obesity, smoking, contraceptive use, immobility level, history of malignant disease and thrombophilia and the factors of familial predisposition, deep vein thrombosis (DVT)/PE-history, long-distance flying $<1$ week and surgery $<4$ weeks previously, were retrospectively examined in 492 patients with a suspected PE. The data demonstrated a significant risk of PE with contraceptive use, a history of DVT/PE and thrombophilia. The immobility level, surgery $<4$ weeks and long-distance flying $<1$ week previously, as well as family history, malignant disease, obesity and smoking, were not observed to be associated with a significantly higher risk of PE. Contraceptive use and thrombophilia, in addition to a history of DVT/PE, each appear to have a significant predictive value in the context of PE risk stratification. Therefore, patients with a suspected PE, who additionally present with at least one of the aforementioned risk factors, should undergo further diagnostic steps for PE risk stratification, including a low-threshold CT examination, even in the absence of elevated D-dimers.
\end{abstract}

Correspondence to: Dr Joachim Gruettner, Emergency Department, University Medical Center Mannheim, Medical Faculty Mannheim, Heidelberg University, 1-3 Theodor-Kutzer-Ufer, Mannheim, Baden-Württemberg D-68167, Germany

E-mail: joachim.gruettner@umm.de

${ }^{*}$ Contributed equally

Key words: pulmonary embolism, risk factors, emergency department

\section{Introduction}

Acute pulmonary embolism (PE) is a major challenge for emergency facilities. On average, $90 \%$ of all mortalities occur within $2 \mathrm{~h}$ of the onset of the symptoms (1). Therefore, the rapid treatment of fulminant PE is a high priority. The optimization of emergency structures has been demonstrated to significantly reduce the mortality rate from unstable PE (2). The reliable exclusion of PE in hemodynamically stable patients remains an additional problem, since in a number of these patients, the symptoms of PE are barely evident or manifest in an atypical manner. Previous studies have shown that PE has been frequently overlooked as a result, and that the mortality rate in such cases is significantly increased $(3,4)$. The most important diagnostic method in suspected cases of PE is computed tomography (CT) scans of the pulmonary artery $(5,6)$.

In 2008, the European Society of Cardiology (ESC) published guidelines for the diagnosis and management of PE (7). These recommendations were based on risk stratification using scores, such as the Wells and Geneva scores (8-10). The most important consequence of the ESC guidelines was a low-threshold indication for CT to take into account the diagnostic uncertainty, as aforementioned. The clinical significance of various factors within the guidelines has been discussed controversially by certain authors (11). Furthermore, the risk of overdiagnosis via the detection of an increasing number of clinically-unapparent subsegmental PEs is similarly controversial.

Overall, despite the publication of ESC guidelines, there remains uncertainty with regard to the risk assessment of PE. Against this background, patients with a suspected PE who were admitted to the Emergency Department of the University Medical Center Mannheim (Mannheim, Germany) in 2010 and 2011 were retrospectively studied and the collected data were published in a previous study (12). The focus of that study was on the incidence rate of PE in cases with low-threshold CT indications and the significance of the laboratory diagnostic markers, troponin and D-dimers (12). Patients with the cardinal symptoms of chest pain, dyspnoea and syncope were included, since these symptoms have been frequently observed in patients with a PE in previous studies (13-17).

The aim of the present study was to address the significance of PE risk factors in the patient collective, as aforementioned. 
In terms of the risk profile of PE, various factors have been proposed by previous studies, including a prior PE or a history of deep vein thrombosis (DVT), obesity, smoking, use of oral contraceptives, thrombophilia, recent long distance flying, malignant disease and immobilization (18-21). However, in general, the patient collectives studied have been small and of heterogeneous quality. Therefore, accurate evaluation of the risk profile in cases suspected of a PE may be important in the context of the current ESC recommendations and in light of the uncertainties described.

\section{Materials and methods}

Study population. In the study, the medical records of 492 patients who were admitted to the Emergency Department of the University Hospital of Mannheim in the period between April 2010 and July 2011 were retrospectively analyzed. Due to the retrospective nature of the study protocol, the institutional ethical review board of the University Medical Center Mannheim waived the requirement for informed patient consent. All the patients presented with cardinal symptoms of chest pain, dyspnoea or syncope, and tested positive for the D-dimer test for PE exclusion. The patients were hemodynamically stable and met the criteria of a low or moderate probability of PE, according to the Wells score (10).

The specific risk profile of all the patients was documented following their admission to the Emergency Department. In the documentation, a number of factors, including obesity, smoking, use of contraceptives, immobility level, history of malignant disease and thrombophilia, family history, history of DVT/PE, long-distance flying $<1$ week prior to admission and surgery during the four preceding weeks, were recorded.

Furthermore, the diagnosis standard comprised a physical examination and a 12-lead electrocardiography (GE MAC 1200 ST; GE Healthcare, Freiburg, Germany). As laboratory risk markers, the levels of D-dimers (TINA-quant; Roche Diagnostics, Mannheim, Germany), as a parameter of acute coagulation activation, and high-sensitivity troponin (Siemens Healthcare Diagnostics, Eschborn, Germany), a marker of cardiac cell death, were determined. In the case of the presence of any of the described cardinal symptoms in combination with a positive D-dimer test, a pulmonary artery CT examination (Somatom Emotion, Siemens Medical Solutions, Forchheim, Germany) was performed. CT was considered a contraindication for pregnant women and patients with contrast medium allergy, higher-grade renal insufficiency (creatinine level of $>1.5 \mathrm{mg} / \mathrm{dl}$ ) or hyperthyroidism, as well as in cases of ongoing metformin therapy. In patients with these contraindications, no acute CT examination was performed.

Statistical analysis. For statistical analysis, JMP 9.0.0 (SAS Institute, Inc., Cary, NC, USA), MedCalc 12.7.0 (MedCalc Software bvba, Ostend, Belgium) and Forest Plot Viewer 1.0 (National Institute of Environmental Health Sciences, Durham, NC, USA) software packages were used. Nominal variables are depicted as numbers (percentages). For each continuous variable, the Shapiro-Wilk test was applied to assess for Gaussian distribution of the data. Parametric and nonparametric continuous variables are expressed as the mean \pm standard deviation and as the median ( $1^{\text {st }}$ and $3^{\text {rd }}$ quartiles), respectively. Furthermore,
Table I. Baseline characteristics of 492 patients with a suspected pulmonary embolism.

\begin{tabular}{lc} 
Characteristic & All patients (n= \\
\hline Age, years & \\
Mean \pm SD & $68 \pm 17$ \\
Range & $19-105$ \\
Gender, $\mathrm{n}(\%)$ & \\
Male & $218(44)$ \\
Female & $274(56)$ \\
Symptoms, $\mathrm{n}(\%)$ & \\
Chest pain & $257(52)$ \\
Dyspnoea & $281(57)$ \\
Syncope & $81(16)$ \\
Heart rate, bpm & \\
Mean \pm SD & $86 \pm 22$ \\
Range & $49-175$ \\
Systolic blood pressure, $\mathrm{mmHg}$ & \\
Mean \pm SD & $147 \pm 29$ \\
Range & $90-290$ \\
Diastolic blood pressure, $\mathrm{mmHg}$ & \\
Mean \pm SD & $80 \pm 15$ \\
Range & $40-150$ \\
Oxygen saturation, $\%$ & \\
Mean \pm SD & $96 \pm 4$ \\
Range & $70-100$
\end{tabular}

$\mathrm{SD}$, standard deviation.

value ranges (minimum and maximum) were presented. As a measure for the quantitative estimation of the risk of PE in cases with the presence of a risk factor, the odds ratio (OR) and the $95 \%$ confidence interval (CI) were calculated. A value of $\mathrm{P}<0.05$, based on two-sided significance testing, was considered to indicate a statistically significant difference.

\section{Results}

Patient characteristics. Baseline characteristics of the patients are summarized in Table I. The specific risk profiles of the 492 patients with a suspected PE, including the number and percentage of patients (n, \%) and the single risk factor ORs for the presence of PE, are listed in Table II. As shown in Fig. 1, the ORs were compared in the form of a Forest plot graph. The risk of $\mathrm{PE}$ was significantly increased with each of the following factors: Thrombophilia (OR, 11.5, $\mathrm{P}=0.008)$, use of contraceptives $(\mathrm{OR}, 4.9, \mathrm{P}=0.03)$ and history of DVT/PE (OR, 3.08, $\mathrm{P}=0.003)$. Surgery $<4$ weeks previously $(\mathrm{OR}, 1.58, \mathrm{P}=0.42)$, immobility level (OR, 2.84, $\mathrm{P}=0.13)$ and long-distance flying $<1$ week previously (OR, 3.78, $\mathrm{P}=0.13$ ), as well as family history of DVT/PE (OR, 5.03, $\mathrm{P}=0.08$ ), neoplastic disease (OR, 1.11, $\mathrm{P}=0.82)$ and obesity $(\mathrm{OR}, 1.22, \mathrm{P}=0.64)$ were shown to increase the risk of PE; however, the difference was not statistically significant. In the comprised collective, smoking was not found to be associated with an increased risk of $\mathrm{PE}(\mathrm{OR}, 0.73, \mathrm{P}=0.47)$. 
Table II. Risk factors in patients $(\mathrm{n}=492)$ with a suspected PE.

\begin{tabular}{lccc}
\hline Risk factor & Cases, $\mathrm{n}(\%)$ & OR & 95\% CI \\
\hline Obesity & $50(10.2)$ & 1.22 & $0.52-85$ \\
Smoking & $74(15.0)$ & 0.73 & $0.32-1.69$ \\
Oral contraceptives & $8(1.6)$ & 4.90 & $1.11-21.59$ \\
Tumor diseases & $46(9.3)$ & 1.11 & $0.45-2.75$ \\
History of DVT/PE & $41(8.3)$ & 3.08 & $1.45-6.54$ \\
Family history of DVT/PE & $5(1.0)$ & 5.03 & $0.82-30.74$ \\
Thrombophilia & $5(1.0)$ & 11.50 & $1.89-70.60$ \\
Long distance flight $<1$ week & $6(1.2)$ & 3.78 & $0.67-21.01$ \\
Immobility & $11(2.2)$ & 2.84 & $0.73-11.04$ \\
Surgery <4 weeks & $23(4.7)$ & 1.58 & $0.003^{\mathrm{a}}$ \\
\hline
\end{tabular}

${ }^{\mathrm{a}} \mathrm{P}<0.05$ which demonstrates a significant association between the risk factor and an increased risk of PE. DVT, deep vein thrombosis; PE, pulmonary embolism; OR, odds ratio; CI, confidence interval.

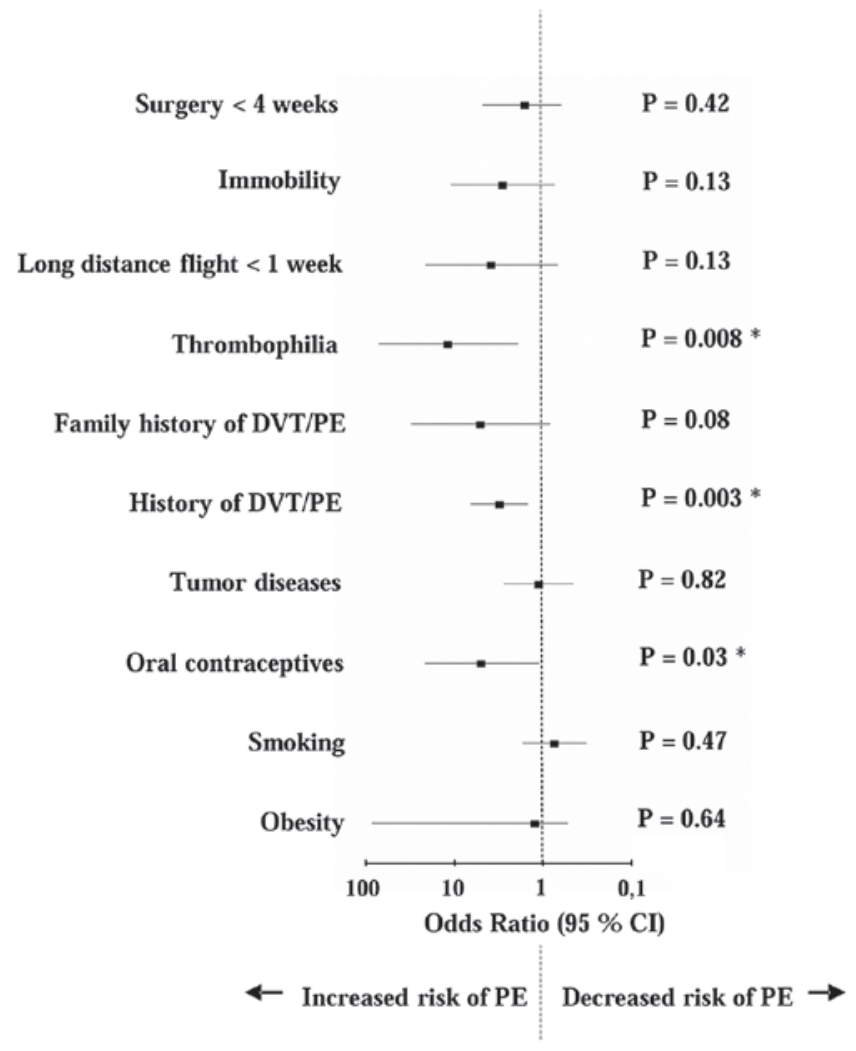

Figure 1. Forest plot demonstrating the odds ratios for the risk factors of patients $(\mathrm{n}=492)$ with a suspected $\mathrm{PE}$. ${ }^{*} \mathrm{P}<0.05$ which demonstrates a significant association between the risk factor and an increased risk of PE. PE, pulmonary embolism; DVT, deep vein thrombosis; CI, confidence interval.

\section{Discussion}

In hemodynamically stable patients, the optimal workflow for the exclusion of a PE remains subject to uncertainty. The ESC guidelines for PE published in 2008 took this into account by recommending a low-threshold $\mathrm{CT}$ indicator for patients with a suspected PE. However, this strategy is considered controversially with regard to the possibility of overdiagnosis of patients, such as by the detection of unapparent subsegmental PEs. Therefore, relevant criteria for further optimization of the risk assessment of these patients is required.

Traditionally, risk factors play an important role in the diagnostic workup of patients with cardiovascular disease. In acute coronary syndrome, for example, the risk factors of coronary heart disease are mapped by relevant scores, such as the Thrombolysis In Myocardial Infarction score (criterion, $\geq 3$ risk factors) (22). Similarly, with regard to PE, a number of risk factors are known, as previously described. However, the Wells score considers only the factors of immobilization or surgery $<4$ weeks previously, prior history of DVT or PE and malignant disease $<6$ months previously (10). In addition, the Geneva score only includes the criteria of a prior history of DVT or PE, surgery or fracture $<4$ weeks previously and active malignancy (9). Other parameters, including obesity, smoking, use of oral contraceptives, thrombophilia and recent long distance flying, are described in the literature; however, are not included into the scores recommended by the ESC (18-21). Therefore, the predictive value of considering these risk factors in the context of modern PE diagnostics is yet to be determined.

The aim of the present study was to re-evaluate the risk factors of $\mathrm{PE}$ in their entirety, and particularly in synopsis with the ESC recommendations published in 2008 and updated in 2014. The current study met the low-threshold indicators for CT, as demanded by the ESC, and thus the modern requirements of PE diagnosis. The results of the present study demonstrated a significant risk of PE for the criterion of contraceptive use and a highly significant risk of PE for the parameters of a history of DVT/PE and thrombophilia. History of DVT/PE is included in the two previously mentioned scoring systems within the ESC guidelines. By contrast, the factors of contraceptive use and thrombophilia are not included in the Wells or Geneva scores. This diagnostic deficiency is, however, difficult to assess. The association between oral contraceptives and PE is established, although only a limited number of studies address the issue $(20,23)$. Of these, the study by Lauque et al comprised only 11 case reports and is not recent (20). Significantly more 
data exist with regard to the association between DVT and contraceptive use. A meta-analysis by Manzoli et al included a considerable number of studies and confirmed a significantly increased risk of thrombosis with oral contraceptive use (23). Despite the wealth of data, the results concerning DVT cannot be transferred directly to PE. Nevertheless, the association between oral contraceptives and PE remains undisputed. With regard to the association between thrombophilia and $\mathrm{PE}$, the data of the Lauque et al study are similarly weak. Data from a large retrospective study by $\mathrm{Wu}$ et al indicated a significantly increased risk of PE associated with different thrombophilia subgroups; the risk increased further upon concomitant intake of contraceptives (24). Thus, the risk factors of contraceptive use and thrombophilia, in addition to a history of DVT/PE, are of significant importance in the context of PE.

In conclusion, the results of the present study demonstrate that emphasis should be focused on the risk factors of contraceptive use, thrombophilia and a history of DVT/PE, in addition to previously used diagnostic parameters, such as D-dimer testing, in the overall assessment of PE risk. Patients with a suspected PE and at least one of the aforementioned risk factors should be referred for CT diagnosis even in the absence of elevated levels of D-dimers, since a number of PE cases that tested negative for D-dimer have been reported (25).

\section{References}

1. Walther A and Böttiger BW: Pulmonary embolism. Wien Med Wochenschr 158: 610-614, 2008 (In German).

2. Horlander KT, Mannino DM and Leeper KV: Pulmonary embolism mortality in the United States, 1979-1998: an analysis using multiple-cause mortality data. Arch Intern Med 163: 1711-1717, 2003.

3. Goldhaber SZ: Pulmonary embolism. Lancet 363: 1295-1305, 2004.

4. Kline JA, Hernandez-Nino J, Jones AE, Rose GA, Norton HJ and Camargo CA Jr: Prospective study of the clinical features and outcomes of emergency department patients with delayed diagnosis of pulmonary embolism. Acad Emerg Med 14: 592-598, 2007

5. Mullins MD, Becker DM, Hagspiel KD and Philbrick JT: The role of spiral volumetric computed tomography in the diagnosis of pulmonary embolism. Arch Intern Med 160 293-298, 2000.

6. Perrier A, Howarth N, Didier D, et al: Performance of helical computed tomography in unselected outpatients with suspected pulmonary embolism. Ann Intern Med 135: 88-97, 2001.

7. Torbicki A, Perrier A, Konstantinides S, et al; ESC Committee for Practice Guidelines (CPG): Guidelines on the diagnosis and management of acute pulmonary embolism: the Task Force for the Diagnosis and Management of Acute Pulmonary Embolism of the European Society of Cardiology (ESC). Eur Heart J 29: 2276-2315, 2008.
8. Douma RA, Mos IC, Erkens PM, et al: Performance of 4 clinical decision rules in the diagnostic management of acute pulmonary embolism: a prospective cohort study. Ann Intern Med 154: 709-718, 2011.

9. Le Gal G, Righini M, Roy PM, Sanchez O, Aujesky D, Bounameaux $\mathrm{H}$ and Perrier A: Prediction of pulmonary embolism in the emergency department: the revised Geneva score. Ann Intern Med 144: 165-171, 2006.

10. Wells PS, Anderson DR, Rodger M, et al: Derivation of a simple clinical model to categorize patients probability of pulmonary embolism: increasing the models utility with the SimpliRED D-dimer. Thromb Haemost 83: 416-420, 2000.

11. Young MD, Daniels AH, Evangelista PT, et al: Predicting pulmonary embolus in orthopedic trauma patients using the wells score. Orthopedics 36: e642-e647, 2013.

12. Gruettner J, Walter T, Bolte M, Haghi D, Sudarski S and Henzler T: Incidence of pulmonary embolism in an emergency department cohort evaluated with a simple symptom-based diagnostic algorithm. In Vivo 27: 215-220, 2013.

13. Calvo-Romero JM, Pérez-Miranda M and Bureo-Dacal P: Syncope in acute pulmonary embolism. Eur J Emerg Med 11: 208-209, 2004.

14. Castelli R, Tarsia P, Tantardini C, Pantaleo G, Guariglia A and Porro F: Syncope in patients with pulmonary embolism: comparison between patients with syncope as the presenting symptom of pulmonary embolism and patients with pulmonary embolism without syncope. Vasc Med 8: 257-261, 2003.

15. Koutkia P and Wachtel TJ: Pulmonary embolism presenting as syncope: case report and review of the literature. Heart Lung 28: 342-347, 1999.

16. Miniati M, Prediletto R, Formichi B, et al: Accuracy of clinical assessment in the diagnosis of pulmonary embolism. Am J Respir Crit Care Med 159: 864-871, 1999.

17. Wolfe TR and Allen TL: Syncope as an emergency department presentation of pulmonary embolism. J Emerg Med 16: 27-31, 1998.

18. Goldhaber SZ, Grodstein F, Stampfer MJ, et al: A prospective study of risk factors for pulmonary embolism in women. JAMA 277: 642-645, 1997.

19. Kline JA and Miller DW: Risk stratification for acute pulmonary embolism. J Natl Compr Canc Netw 9: 800-810, 2011

20. Lauque D, Mazières J, Rouzaud P, et al: Pulmonary embolism in patients using estrogen-progestagen contraceptives. Presse Med 27: 1566-1569, 1998 (In French).

21. Lehmann R, Suess C, Leus M, et al: Incidence, clinical characteristics and long-term prognosis of travel-associated pulmonary embolism. Eur Heart J 30: 233-241, 2009.

22. Antman EM, Cohen M, Bernink PJ, et al: The TIMI risk score for unstable angina/non-ST elevation MI: A method for prognostication and therapeutic decision making. JAMA 284: 835-842, 2000.

23. Manzoli L, De Vito C, Marzuillo C, Boccia A and Villari P: Oral contraceptives and venous thromboembolism: a systematic review and meta-analysis. Drug Saf 35: 191-205, 2012.

24. Wu O, Robertson L, Twaddle S, et al: Screening for thrombophilia in high-risk situations: systematic review and cost-effectiveness analysis. The thrombosis: risk and economic assessment of thrombophilia screening (TREATS) study. Health Technol Assess 10: 100-110, 2006.

25. Breen ME, Dorfman M and Chan SB: Pulmonary embolism despite negative ELISA D-dimer: a case report. J Emerg Med 37: 290-292, 2009. 